\title{
Autologous BCMA-targeted CAR T Cells LCAR-B4822M
}

National Cancer Institute

\section{Source}

National Cancer Institute. Autologous BCMA-targeted CART Cells LCAR-B4822M. NCI

Thesaurus. Code C162506.

A preparation of autologous peripheral blood T-lymphocytes (PBT Ls) that have been genetically modified to express a chimeric antigen receptor (CAR) specific for the B-cell maturation antigen (BCMA; tumor necrosis factor receptor superfamily member 17; TNFRSF17), with potential immunostimulating and antineoplastic activities. Upon administration, autologous BCMA-targeted CAR T-cells LCAR-B4822M specifically recognize and kill BCMA-expressing tumor cells. BCMA, a tumor specific antigen and a receptor for both a proliferation-inducing ligand (APRIL) and B-cell activating factor (BAFF), is a member of the tumor necrosis factor (TNF) receptor superfamily and plays a key role in plasma cell survival; it is found on the surfaces of plasma cells and overexpressed on malignant plasma cells. 